\title{
Generalized Reflexive and Generalized Antireflexive Solutions to a System of Matrix Equations
}

\author{
Yong Lin ${ }^{1,2}$ and Qing-Wen Wang ${ }^{1}$ \\ ${ }^{1}$ Department of Mathematics, Shanghai University, Shanghai 200444, China \\ ${ }^{2}$ School of Mathematics and Statistics, Suzhou University, Suzhou 234000, China \\ Correspondence should be addressed to Yong Lin; ly_lmc@126.com
}

Received 23 March 2013; Accepted 30 December 2013; Published 23 February 2014

Academic Editor: Jinyun Yuan

Copyright ( 2014 Y. Lin and Q.-W. Wang. This is an open access article distributed under the Creative Commons Attribution License, which permits unrestricted use, distribution, and reproduction in any medium, provided the original work is properly cited.

Two efficient iterative algorithms are presented to solve a system of matrix equations $A_{1} X_{1} B_{1}+A_{2} X_{2} B_{2}=E, C_{1} X_{1} D_{1}+$ $C_{2} X_{2} D_{2}=F$ over generalized reflexive and generalized antireflexive matrices. By the algorithms, the least norm generalized reflexive (antireflexive) solutions and the unique optimal approximation generalized reflexive (antireflexive) solutions to the system can be obtained, too. For any initial value, it is proved that the iterative solutions obtained by the proposed algorithms converge to their true values. The given numerical examples demonstrate that the iterative algorithms are efficient.

\section{Introduction}

Throughout the paper, we denote the set of all $m \times n$ real matrices by $R^{m \times n}$, the transpose matrix of $A$ by $A^{T}$, the identity matrix of order $n$ by $I_{n}$, the Kronecker product of $A$ and $B$ by $A \otimes B$, the $m n \times 1$ vector formed by the vertical concatenation of the respective columns of a matrix $A \in R^{m \times n}$ by $\operatorname{vec}(A)$, the trace of a matrix $A$ by $\operatorname{tr}(A)$, and the Frobenius norm of a matrix $A$ by $\|A\|$, where $\|A\|=\sqrt{\operatorname{tr}\left(A^{T} A\right)}$. Let $P \in R^{m \times m}$ and $Q \in R^{n \times n}$ be two real generalized reflection matrices, that is; $P^{T}=P, P^{2}=I_{m}$ and $Q^{T}=\mathrm{Q}$, $Q^{2}=I_{n}$. A matrix $A \in R^{m \times n}$ is called generalized reflexive (antireflexive) matrix with respect to the matrix pair $(P, Q)$ if $A=P A Q(A=-P A Q)$. The set of all $m \times n$ real generalized reflexive (generalized antireflexive) matrices with respect to matrix pair $(P, Q)$ is denoted by $R_{r}^{m \times n}(P, Q)\left(R_{a}^{m \times n}(P, Q)\right)$. The generalized reflexive and generalized antireflexive matrices have been widely used in engineering, scientific computations, and various other fields.

The linear matrix equation pair $A_{1} X_{1} B_{1}+A_{2} X_{2} B_{2}=E$, $C_{1} X_{1} D_{1}+C_{2} X_{2} D_{2}=F$ is encountered in many systems and control applications. In this paper, we consider it with real generalized reflexive and generalized antireflexive constraint on the solutions. Let $T_{1}, T_{2}, T_{3}$, and $T_{4}$ be four real generalized reflection matrices; the following four problems are studied.

Problem 1. For given matrices $A_{1} \in R^{p \times k}, A_{2} \in R^{p \times m}, B_{1} \in$ $R^{r \times q}, B_{2} \in R^{n \times q}, C_{1} \in R^{s \times k}, C_{2} \in R^{s \times m}, D_{1} \in R^{r \times t}, D_{2} \in R^{n \times t}$, $E \in R^{p \times q}$, and $F \in R^{s \times t}$, find $X_{1} \in S_{1} \subset R_{r}^{k \times r}\left(T_{1}, T_{2}\right)$ and $X_{2} \in S_{2} \subset R_{r}^{m \times n}\left(T_{3}, T_{4}\right)$ such that

$$
\begin{aligned}
& A_{1} X_{1} B_{1}+A_{2} X_{2} B_{2}=E, \\
& C_{1} X_{1} D_{1}+C_{2} X_{2} D_{2}=F .
\end{aligned}
$$

Problem 2. When Problem 1 is consistent, let $S_{r}$ denote the set of its solutions. For the given matrices $\widetilde{X}_{1} \in R^{k \times r}, \widetilde{X}_{2} \in R^{m \times n}$, find $\left\{\widehat{X}_{1}, \widehat{X}_{2}\right\} \in S_{r}$ such that

$$
\begin{aligned}
& \left\|\widehat{X}_{1}-\widetilde{X}_{1}\right\|^{2}+\left\|\widehat{X}_{2}-\widetilde{X}_{2}\right\|^{2} \\
& =\min _{\left\{X_{1}, X_{2}\right\} \in S_{r}}\left(\left\|X_{1}-\widetilde{X}_{1}\right\|^{2}+\left\|X_{2}-\widetilde{X}_{2}\right\|^{2}\right) .
\end{aligned}
$$

Problem 3. For given matrices $A_{1} \in R^{p \times k}, A_{2} \in R^{p \times m}, B_{1} \in$ $R^{r \times q}, B_{2} \in R^{n \times q}, C_{1} \in R^{s \times k}, C_{2} \in R^{s \times m}, D_{1} \in R^{r \times t}, D_{2} \in R^{n \times t}$, 
$E \in R^{p \times q}$ and $F \in R^{s \times t}$, find $X_{1} \in S_{3} \subset R_{a}^{k \times r}\left(T_{3}, T_{4}\right)$ and $X_{2} \in S_{4} \subset R_{a}^{m \times n}\left(T_{3}, T_{4}\right)$ such that

$$
\begin{aligned}
& A_{1} X_{1} B_{1}+A_{2} X_{2} B_{2}=E, \\
& C_{1} X_{1} D_{1}+C_{2} X_{2} D_{2}=F .
\end{aligned}
$$

Problem 4. When Problem 3 is consistent, let $S_{a}$ denote the set of its solutions. For the given matrices $\widetilde{X}_{1} \in R^{k \times r}, \widetilde{X}_{2} \in R^{m \times n}$, find $\left\{\widehat{X}_{1}, \widehat{X}_{2}\right\} \in S_{a}$ such that

$$
\begin{aligned}
\| \widehat{X}_{1} & -\widetilde{X}_{1}\left\|^{2}+\right\| \widehat{X}_{2}-\widetilde{X}_{2} \|^{2} \\
& =\min _{\left\{X_{1}, X_{2}\right\} \in S_{a}}\left(\left\|X_{1}-\widetilde{X}_{1}\right\|^{2}+\left\|X_{2}-\widetilde{X}_{2}\right\|^{2}\right) .
\end{aligned}
$$

Problem 2 (4) is to find the optimal approximation generalized reflexive (antireflexive) solution to the given generalized reflexive (antireflexive) matrices $\widetilde{X}_{1}$ and $\widetilde{X}_{2}$ in the solution set of Problem 1 (3); it is occurs frequently in experiment design (see, e.g., [1]). In recent years, the matrix optimal approximation problem has been studied extensively (e.g., [2-12]).

Research on solving matrix equation pair has been actively ongoing for the last 30 years or more. For instance, Mitra [13] gave conditions for the existence of a solution and a representation of the general common solution to $A X B=E$, $C X D=F$. Shinozaki and Sibuya [14] and van der Woude [15] discussed conditions for the existence of a common solution to $A X B=E, C X D=F$. Navarra et al. [5] derived sufficient and necessary conditions for the existence of a common solution to $A X B=E, C X D=F$. Yuan [11] obtained an analytical expression of the least-squares solutions of $A X B=E, C X D=$ $F$ by using the generalized singular value decomposition (GSVD) of matrices. Dehghan and Hajarian [32] presented some examples to show a motivation for studying the general coupled matrix equations $\sum_{j=1}^{l} A_{i j} X_{j} B_{i j}=C_{i}, i=1,2, \ldots, l$, and they [17] constructed an iterative algorithm to solve the general coupled matrix equations $\sum_{j=1}^{p} A_{i j} X_{j} B_{i j}=M_{i}, i=$ $1,2, \ldots, p$. Wang $[18,19]$ gave the centrosymmetric solution to the system of quaternion matrix equations $A_{1} X=C_{1}$, $A_{3} X B_{3}=C_{3}$. Wang [16] also solved a system of matrix equations over arbitrary regular rings with identity.

Recently, some finite iterative algorithms have also been developed to solve matrix equations. Ding et al. [20-23] studied the iterative solutions of matrix equations $A X B=$ $F$ and $A_{i} X B_{i}=F_{i}$, generalized Sylvester matrix equations $A X B+C X D=F$ and $A X B+C X^{T} D=F$. They presented gradient based and least-squares based iterative algorithms for the solution. Duan et al. [24-27] considered iterative method for some coupled linear matrix equations. Deng et al. [28] studied the consistent conditions and the general expressions about the Hermitian solutions of the matrix equations $(A X, X B)=(C, D)$ and designed an iterative method for its Hermitian minimum norm solutions. Li and $\mathrm{Wu}$ [29] gave symmetric and skew-antisymmetric solutions to certain matrix equations $A_{1} X=C_{1}, X B_{3}=C_{3}$ over the real quaternion algebra $H$. For more studies on iterative algorithms on coupled matrix equations, we refer to $[3,8-$ 10, 30-36]. Peng et al. [12] presented iterative methods to obtain the symmetric solutions of $A X B=E, C X D=F$. Sheng and Chen [7] presented a finite iterative method for solving $A X B=E, C X D=F$. Liao and Lei [37] presented an analytical expression of the least-squares solution and an algorithm for $A X B=E, C X D=F$ with the minimum norm. Peng et al. [6] presented an algorithm for the least-squares reflexive solution. Dehghan and Hajarian [2] presented an iterative algorithm for solving a pair of matrix equations $A X B=E, C X D=F$ over generalized matrices; they [31] also presented an algorithm for solving a pair of matrix equations $A X B+C Y D=J, E X F+G Y H=K$ over generalized reflexive (antireflexive) matrices. Cai and Chen [38] presented an iterative algorithm for the least-squares bisymmetric solutions of the matrix equations $A X B=E$, $C X D=F$. Yin and Huang [39] presented an iterative algorithm to solve the least-squares generalized reflexive solutions of the matrix equations $A X B=E, C X D=F$. Lin and Wang [40] presented an iterative algorithm to solve a system of linear matrix equations $A_{1} X_{1} B_{1}+A_{2} X_{2} B_{2}=E$, $C_{1} X_{1} D_{1}+C_{2} X_{2} D_{2}=F$ with real matrices $X_{1}$ and $X_{2}$.

However, to our knowledge, there has been little information on finding the solutions to the Problems 1-4 by iterative algorithm. In this paper, two efficient iterative algorithms are presented to solve the Problems 1-4. The suggested iterative algorithms automatically determine the solvability of equations pair (1) with the constraint. When the pair of equations is consistent, then, for any initial generalized reflexive (antireflexive) matrices $X_{1}^{1}$ and $X_{2}^{1}$, the solution can be obtained in the absence of round errors, and the least norm solution can be obtained by choosing a special kind of initial matrix. In addition, the unique optimal approximation solution pair $\widehat{X}_{1}, \widehat{X}_{2}$ to given matrix pair $\widetilde{X}_{1}, \widetilde{X}_{2}$ in Frobenius norm can be obtained by finding the least norm solution of a new pair of matrix equations $A_{1} \bar{X}_{1} B_{1}+A_{2} \bar{X}_{2} B_{2}=\bar{E}$, $C_{1} \bar{X}_{1} D_{1}+C_{2} \bar{X}_{2} D_{2}=\bar{F}$, where $\bar{X}_{i}=X_{i}-\bar{X}_{i},(i=1,2)$, $\bar{E}=E-A_{1} \widetilde{X}_{1} B_{1}+A_{2} \widetilde{X}_{2} B_{2}, \bar{F}=F-C_{1} \widetilde{X}_{1} D_{1}+C_{2} \widetilde{X}_{2} D_{2}$. The given numerical examples demonstrate that our iterative algorithms are efficient. In particular, when the numbers of the parameter matrices $A_{1}, A_{2}, B_{1}, B_{2}, C_{1}, C_{2}, D_{1}$, and $D_{2}$ are large, our algorithms are efficient as well while the algorithm of [31] is not convergent. That is, our algorithms have merits of good numerical stability and ease to program.

The rest of this paper is outlined as follows. In Section 2, we first propose an efficient iterative algorithm for solving Problems 1 and 2; then we give some properties of this iterative algorithm. We show that the algorithm can obtain a solution group for any (special) initial generalized reflexive matrix group in the absence of round-off errors. In Section 3, Problems 3 and 4 are solved similarly. In Section 4 numerical examples are given to illustrate that our algorithms are quite efficient.

\section{Iterative Algorithm for Solving Problems 1 and 2}

In this section, we present an iterative algorithm for solving Problems 1 and 2. 
Algorithm 5. (1) Input matrices $A_{1} \in R^{p \times k}, A_{2} \in R^{p \times m}, B_{1} \in$ $R^{r \times q}, B_{2} \in R^{n \times q}, C_{1} \in R^{s \times k}, C_{2} \in R^{s \times m}, D_{1} \in R^{r \times t}, D_{2} \in R^{n \times t}$, $E \in R^{p \times q}, F \in R^{s \times t}, X_{1}^{1} \in R_{r}^{k \times r}$, and $X_{2}^{1} \in R_{r}^{m \times n}$ (where $X_{1}^{1}, X_{2}^{1}$ are any initial generalized reflexive matrices).

(2) Calculate

$$
\begin{gathered}
E^{1}=E ; \quad F^{1}=F ; \\
Q_{1}^{1}=A_{1}^{T} E^{1} B_{1}^{T}+C_{1}^{T} F^{1} D_{1}^{T} ; \quad Q_{2}^{1}=A_{2}^{T} E^{1} B_{2}^{T}+C_{2}^{T} F^{1} D_{2}^{T} ; \\
P_{1}^{1}=Q_{1}^{1}+T_{1} Q_{1}^{1} T_{2} ; \quad P_{2}^{1}=Q_{2}^{1}+T_{3} Q_{2}^{1} T_{4} ; \\
\beta^{1}=\left(\operatorname{tr}\left[\left(E^{1}\right)^{T}\left(A_{1} P_{1}^{1} B_{1}+A_{2} P_{2}^{1} B_{2}\right)\right]\right. \\
\left.+\operatorname{tr}\left[\left(F^{1}\right)^{T}\left(C_{1} P_{1}^{1} D_{1}+C_{2} P_{2}^{1} D_{2}\right)\right]\right) \\
\times\left(\left\|A_{1} P_{1}^{1} B_{1}+A_{2} P_{2}^{1} B_{2}\right\|^{2}+\left\|C_{1} P_{1}^{1} D_{1}+C_{2} P_{2}^{1} D_{2}\right\|^{2}\right)^{-1} ; \\
\Delta X_{1}^{1}=\beta^{1} P_{1}^{1} ; \quad \Delta X_{2}^{1}=\beta^{1} P_{2}^{1} ; \quad k=1 .
\end{gathered}
$$

(3) If $\Delta X=\operatorname{diag}\left(\Delta X_{1}^{k}, \Delta X_{2}^{k}\right)=0(k=1,2, \ldots)$, then stop. Otherwise,

$$
\begin{aligned}
& X_{1}^{k+1}=X_{1}^{k}+\Delta X_{1}^{k} ; \\
& X_{2}^{k+1}=X_{2}^{k}+\Delta X_{2}^{k} .
\end{aligned}
$$

(4) Calculate

$$
\begin{gathered}
E^{k+1}=E^{k}-\left(A_{1} \Delta X_{1}^{k} B_{1}+A_{2} \Delta X_{2}^{k} B_{2}\right) ; \\
F^{k+1}=F^{k}-\left(C_{1} \Delta X_{1}^{k} D_{1}+C_{2} \Delta X_{2}^{k} D_{2}\right) ; \\
Q_{1}^{k+1}=A_{1}^{T} E^{k+1} B_{1}^{T}+C_{1}^{T} F^{k+1} D_{1}^{T} ; \\
Q_{2}^{k+1}=A_{2}^{T} E^{k+1} B_{2}^{T}+C_{2}^{T} F^{k+1} D_{2}^{T} ; \\
P_{1}^{k+1}=Q_{1}^{k+1}+T_{1} Q_{1}^{k+1} T_{2} ; \quad P_{2}^{k+1}=Q_{2}^{k+1}+T_{3} Q_{2}^{k+1} T_{4} ; \\
\beta^{k+1}=\left(\operatorname{tr}\left[\left(E^{k+1}\right)^{T}\left(A_{1} P_{1}^{k+1} B_{1}+A_{2} P_{2}^{k+1} B_{2}\right)\right]\right. \\
\left.+\operatorname{tr}\left[\left(F^{k+1}\right)^{T}\left(C_{1} P_{1}^{k+1} D_{1}+C_{2} P_{2}^{k+1} D_{2}\right)\right]\right) \\
\times\left(\left\|A_{1} P_{1}^{k+1} B_{1}+A_{2} P_{2}^{k+1} B_{2}\right\|^{2}\right. \\
\left.+\left\|C_{1} P_{1}^{k+1} D_{1}+C_{2} P_{2}^{k+1} D_{2}\right\|^{2}\right)^{-1} ; \\
\Delta X_{1}^{k+1}=\beta^{k+1} P_{1}^{k+1} ; \quad \Delta X_{2}^{k+1}=\beta^{k+1} P_{2}^{k+1} ; \\
k=k+1 .
\end{gathered}
$$

Go to (3).

Lemma 6. In Algorithm 5, the choice of $\beta^{k}$ makes $\left\|\operatorname{diag}\left(E^{k+1}, F^{k+1}\right)\right\|$ reach a minimum and $\operatorname{diag}\left(E^{k+1}, F^{k+1}\right)$ and $\operatorname{diag}\left(A_{1} \Delta X_{1}^{k} B_{1}+A_{2} \Delta X_{2}^{k} B_{2}, C_{1} \Delta X_{1}^{k} D_{1}+C_{2} \Delta X_{2}^{k} D_{2}\right)$ orthogonal to each other.

Proof. From Algorithm 5, we have

$$
\begin{gathered}
\left\|\operatorname{diag}\left(E^{k+1}, F^{k+1}\right)\right\|^{2} \\
=\| \operatorname{diag}\left(E^{k}-\left(A_{1} \Delta X_{1}^{k} B_{1}+A_{2} \Delta X_{2}^{k} B_{2}\right),\right. \\
\left.F^{k}-\left(C_{1} \Delta X_{1}^{k} D_{1}+C_{2} \Delta X_{2}^{k} D_{2}\right)\right) \|^{2} \\
=\| \operatorname{diag}\left(E^{k}-\left(A_{1} \beta^{k} P_{1}^{k} B_{1}+A_{2} \beta^{k} P_{2}^{k} B_{2}\right),\right. \\
\left.\quad F^{k}-\left(C_{1} \beta^{k} P_{1}^{k} D_{1}+C_{2} \beta^{k} P_{2}^{k} D_{2}\right)\right) \|^{2} \\
=\left\|E^{k}-\left(A_{1} \beta^{k} P_{1}^{k} B_{1}+A_{2} \beta^{k} P_{2}^{k} B_{2}\right)\right\|^{2} \\
+\left\|F^{k}-\left(C_{1} \beta^{k} P_{1}^{k} D_{1}+C_{2} \beta^{k} P_{2}^{k} D_{2}\right)\right\|^{2} \\
=\left\|E^{k}\right\|^{2}+\left\|F^{k}\right\|^{2} \\
-2\left[\operatorname{tr}\left(\left(E^{k}\right)^{T}\left(A_{1} P_{1}^{k} B_{1}+A_{2} P_{2}^{k} B_{2}\right)\right)\right. \\
\left.+\operatorname{tr}\left(\left(F^{k}\right)^{T}\left(C_{1} P_{1}^{k} D_{1}+C_{2} P_{2}^{k} D_{2}\right)\right)\right] \beta^{k} \\
+\left[\left\|A_{1} P_{1}^{k} B_{1}+A_{2} P_{2}^{k} B_{2}\right\|^{2}\right. \\
\left.+\left\|C_{1} P_{1}^{k} D_{1}+C_{2} P_{2}^{k} D_{2}\right\|^{2}\right]\left(\beta^{k}\right)^{2} .
\end{gathered}
$$

From the above, the condition of $\left\|\operatorname{diag}\left(E^{k+1}, F^{k+1}\right)\right\|$ reaching a minimum is

$$
\begin{aligned}
\beta^{k}= & \operatorname{tr}\left[\left(E^{k}\right)^{T}\left(A_{1} P_{1}^{k} B_{1}+A_{2} P_{2}^{k} B_{2}\right)\right] \\
& \left.+\operatorname{tr}\left[\left(F^{k}\right)^{T}\left(C_{1} P_{1}^{k} D_{1}+C_{2} P_{2}^{k} D_{2}\right)\right]\right) \\
& \times\left(\left\|A_{1} P_{1}^{k} B_{1}+A_{2} P_{2}^{k} B_{2}\right\|^{2}+\left\|C_{1} P_{1}^{k} D_{1}+C_{2} P_{2}^{k} D_{2}\right\|^{2}\right)^{-1} .
\end{aligned}
$$

On the other side, if the choice of $\beta^{k}$ makes $\operatorname{diag}\left(E^{k+1}, F^{k+1}\right)$ and $\operatorname{diag}\left(A_{1} \Delta X_{1}^{k} B_{1}+A_{2} \Delta X_{2}^{k} B_{2}, C_{1} \Delta X_{1}^{k} D_{1}+\right.$ $C_{2} \Delta X_{2}^{k} D_{2}$ ) orthogonal to each other, that is, $\operatorname{tr}\left[\operatorname{diag}\left(E^{k+1}, F^{k+1}\right)^{T} \operatorname{diag}\left(A_{1} \Delta X_{1}^{k} B_{1}+A_{2} \Delta X_{2}^{k} B_{2}, C_{1} \Delta X_{1}^{k} D_{1}+\right.\right.$ $\left.\left.C_{2} \Delta X_{2}^{k} D_{2}\right)\right]=0$, we can have the same $\beta^{k}$ as (3).

Theorem 7. Algorithm 5 is bound to be convergent. 
Proof. From Algorithm 5 and Lemma 6 we have

$$
\begin{aligned}
\operatorname{diag} & \left(E^{k}, F^{k}\right) \\
= & \operatorname{diag}\left(E^{k+1}, F^{k+1}\right) \\
& +\operatorname{diag}\left(A_{1} \Delta X_{1}^{k} B_{1}+A_{2} \Delta X_{2}^{k} B_{2}, C_{1} \Delta X_{1}^{k} D_{1}+C_{2} \Delta X_{2}^{k} D_{2}\right) \\
\Longleftrightarrow & \left\|\operatorname{diag}\left(E^{k}, F^{k}\right)\right\|^{2} \\
= & \| \operatorname{diag}\left(E^{k+1}, F^{k+1}\right) \\
& +\operatorname{diag}\left(A_{1} \Delta X_{1}^{k} B_{1}+A_{2} \Delta X_{2}^{k} B_{2},\right. \\
= & \left.\left\|\operatorname{diag}\left(E_{1}^{k+1}, F^{k+1}\right)\right\|_{1}^{k} D_{1}+C_{2} \Delta X_{2}^{k} D_{2}\right) \|^{2} \\
& +\| \operatorname{diag}\left(A_{1} \Delta X_{1}^{k} B_{1}+A_{2} \Delta X_{2}^{k} B_{2},\right. \\
& \left.\quad C_{1} \Delta X_{1}^{k} D_{1}+C_{2} \Delta X_{2}^{k} D_{2}\right) \|^{2}
\end{aligned}
$$

such that

$$
\begin{aligned}
& \left\|\operatorname{diag}\left(E^{k+1}, F^{k+1}\right)\right\|^{2} \\
& =\left\|\operatorname{diag}\left(E^{k}, F^{k}\right)\right\|^{2} \\
& -\| \operatorname{diag}\left(A_{1} \Delta X_{1}^{k} B_{1}+A_{2} \Delta X_{2}^{k} B_{2},\right. \\
& \left.C_{1} \Delta X_{1}^{k} D_{1}+C_{2} \Delta X_{2}^{k} D_{2}\right) \|^{2} \\
& \leq\left\|\operatorname{diag}\left(E^{k}, F^{k}\right)\right\|^{2} .
\end{aligned}
$$

From (11), we know that Algorithm 5 is convergent.

Lemma 8 (see [41]). Suppose that the consistent system of linear equations $M y=b$ has a solution $y_{0} \in R\left(M^{T}\right)$; then $y_{0}$ is the least Frobenius norm solution of the system of linear equations.

Theorem 9. Assume that the system (1) is consistent. Let $X_{1}^{1}=$ $A_{1}^{T} Y B_{1}^{T}+C_{1}^{T} Z D_{1}^{T}+T_{1}\left(A_{1}^{T} Y B_{1}^{T}+C_{1}^{T} Z D_{1}^{T}\right) T_{2}, X_{2}^{1}=A_{2}^{T} Y B_{2}^{T}+$ $C_{2}^{T} Z D_{2}^{T}+T_{3}\left(A_{2}^{T} Y B_{2}^{T}+C_{2}^{T} Z D_{2}^{T}\right) T_{4}$ be initial matrices, where $Y \in R^{p \times q}, Z \in R^{s \times t}$ are any initial matrices, or, especially, $X_{1}^{1}=$ $0, X_{2}^{1}=0$; then the solution generated by Algorithm 5 is the least Frobenius norm solution to (1).
Proof. If (1) is consistent, from $X_{1}^{1}=A_{1}^{T} Y B_{1}^{T}+C_{1}^{T} Z D_{1}^{T}+$ $T_{1}\left(A_{1}^{T} Y B_{1}^{T}+C_{1}^{T} Z D_{1}^{T}\right) T_{2}, X_{2}^{1}=A_{2}^{T} Y B_{2}^{T}+C_{2}^{T} Z D_{2}^{T}+T_{3}\left(A_{2}^{T} Y B_{2}^{T}+\right.$ $\left.C_{2}^{T} Z D_{2}^{T}\right) T_{4}$, using Algorithm 5, we have the generalized reflexive iterative solution pair $X_{1}^{k}, X_{2}^{k}$ of (1) as the following:

$$
\begin{aligned}
X_{1}^{k}= & X_{1}^{k-1}+\Delta X_{1}^{k-1} \\
= & X_{1}^{1}+\Delta X_{1}^{1}+\cdots+\Delta X_{1}^{k-1} \\
= & A_{1}^{T} Y B_{1}^{T}+C_{1}^{T} Z D_{1}^{T}+T_{1}\left(A_{1}^{T} Y B_{1}^{T}+C_{1}^{T} Z D_{1}^{T}\right) T_{2} \\
& +A_{1}^{T}\left[\beta^{1} E^{1}+\cdots+\beta^{k-1} E^{k-1}\right] B_{1}^{T} \\
& +C_{1}^{T}\left[\beta^{1} F^{1}+\cdots+\beta^{k-1} F^{k-1}\right] D_{1}^{T} \\
& +T_{1} A_{1}^{T}\left[\beta^{1} E^{1}+\cdots+\beta^{k-1} E^{k-1}\right] B_{1}^{T} T_{2} \\
& +T_{1} C_{1}^{T}\left[\beta^{1} F^{1}+\cdots+\beta^{k-1} F^{k-1}\right] D_{1}^{T} T_{2} \\
= & A_{1}^{T} M B_{1}^{T}+C_{1}^{T} N D_{1}^{T}+T_{1}\left(A_{1}^{T} M B_{1}^{T}+C_{1}^{T} N D_{1}^{T}\right) T_{2}, \\
X_{2}^{k}= & X_{2}^{k-1}+\Delta X_{2}^{k-1} \\
= & X_{2}^{1}+\Delta X_{2}^{1}+\cdots+\Delta X_{2}^{k-1} \\
= & A_{2}^{T} Y B_{2}^{T}+C_{2}^{T} Z D_{2}^{T}+T_{3}\left(A_{2}^{T} Y B_{2}^{T}+C_{2}^{T} Z D_{2}^{T}\right) T_{4} \\
& +A_{2}^{T}\left[\beta^{1} E^{1}+\cdots+\beta^{k-1} E^{k-1}\right] B_{2}^{T} \\
& +C_{2}^{T}\left[\beta^{1} F^{1}+\cdots+\beta^{k-1} F^{k-1}\right] D_{2}^{T}\left[\beta_{2}^{1} E^{1}+\cdots+\beta^{k-1} E^{k-1}\right] B_{2}^{T} T_{4} \\
& \left.A_{2}^{T} F^{1}+\cdots+\beta_{2}^{k-1} F^{k-1}\right] D_{2}^{T} T_{4}\left(A_{2}^{T} M B_{2}^{T}+C_{2}^{T} N D_{2}^{T}\right) T_{4} .
\end{aligned}
$$

We know that (1) is equivalent to the system

$$
\begin{aligned}
& \left(\begin{array}{cc}
B_{1}^{T} \otimes A_{1} & B_{2}^{T} \otimes A_{2} \\
D_{1}^{T} \otimes C_{1} & D_{2}^{T} \otimes C_{2} \\
\left(T_{2} B_{1}\right)^{T} \otimes\left(A_{1} T_{1}\right) & \left(T_{4} B_{2}\right)^{T} \otimes\left(A_{2} T_{3}\right) \\
\left(T_{2} D_{1}\right)^{T} \otimes\left(C_{1} T_{1}\right) & \left(D_{2} T_{4}\right)^{T} \otimes\left(C_{2} T_{3}\right)
\end{array}\right)\left(\begin{array}{c}
\operatorname{vec}\left(X_{1}\right) \\
\operatorname{vec}\left(X_{2}\right)
\end{array}\right) \\
& =\left(\begin{array}{c}
\operatorname{vec}(E) \\
\operatorname{vec}(F) \\
\operatorname{vec}(E) \\
\operatorname{vec}(F)
\end{array}\right) .
\end{aligned}
$$


From (12) and (13) we have

$$
\begin{aligned}
& \left(\begin{array}{l}
\operatorname{vec}\left(A_{1}^{T} M B_{1}^{T}+C_{1}^{T} N D_{1}^{T}+T_{1}\left(A_{1}^{T} M B_{1}^{T}+C_{1}^{T} N D_{1}^{T}\right) T_{2}\right) \\
\operatorname{vec}\left(A_{2}^{T} M B_{2}^{T}+C_{2}^{T} N D_{2}^{T}+T_{3}\left(A_{2}^{T} M B_{2}^{T}+C_{2}^{T} N D_{2}^{T}\right) T_{4}\right)
\end{array}\right) \\
& =\left(\begin{array}{cccc}
B_{1} \otimes A_{1}^{T} & D_{1} \otimes C_{1}^{T} & \left(T_{2} B_{1}\right) \otimes\left(T_{1} A_{1}^{T}\right) & \left(T_{2} D_{1}\right) \otimes\left(T_{1} C_{1}^{T}\right) \\
B_{2} \otimes A_{2}^{T} & D_{2} \otimes C_{2}^{T} & \left(T_{4} B_{2}\right) \otimes\left(T_{3} A_{2}^{T}\right) & \left(T_{4} D_{2}\right) \otimes\left(T_{3} C_{2}^{T}\right)
\end{array}\right)\left(\begin{array}{c}
\operatorname{vec}(M) \\
\operatorname{vec}(N) \\
\operatorname{vec}(M) \\
\operatorname{vec}(N)
\end{array}\right) \\
& =\left(\begin{array}{cc}
B_{1}^{T} \otimes A_{1} & B_{2}^{T} \otimes A_{2} \\
D_{1}^{T} \otimes C_{1} & D_{2}^{T} \otimes C_{2} \\
\left(B_{1}^{T} T_{2}\right) \otimes\left(A_{1} T_{1}\right) & \left(B_{2}^{T} T_{4}\right) \otimes\left(A_{2} T_{3}\right) \\
\left(D_{1}^{T} T_{2}\right) \otimes\left(C_{1} T_{1}\right) & \left(D_{2}^{T} T_{4}\right) \otimes\left(C_{2} T_{3}\right)
\end{array}\right)^{T}\left(\begin{array}{c}
\operatorname{vec}(M) \\
\operatorname{vec}(N) \\
\operatorname{vec}(M) \\
\operatorname{vec}(N)
\end{array}\right) \\
& \in R\left(\left(\begin{array}{cc}
B_{1}^{T} \otimes A_{1} & B_{2}^{T} \otimes A_{2} \\
D_{1}^{T} \otimes C_{1} & D_{2}^{T} \otimes C_{2} \\
\left(B_{1}^{T} T_{2}\right) \otimes\left(A_{1} T_{1}\right) & \left(B_{2}^{T} T_{4}\right) \otimes\left(A_{2} T_{3}\right) \\
\left(D_{1}^{T} T_{2}\right) \otimes\left(C_{1} T_{1}\right) & \left(D_{2}^{T} T_{4}\right) \otimes\left(C_{2} T_{3}\right)
\end{array}\right)^{T}\right)
\end{aligned}
$$

where $R(*)$ is the column space of matrix $*$.

Considering Lemma 8 , with the initial matrices $X_{1}^{1}=$ $A_{1}^{T} Y B_{1}^{T}+C_{1}^{T} Z D_{1}^{T}+T_{1}\left(A_{1}^{T} Y B_{1}^{T}+C_{1}^{T} Z D_{1}^{T}\right) T_{2}, X_{2}^{1}=A_{2}^{T} Y B_{2}^{T}+$ $C_{2}^{T} Z D_{2}^{T}+T_{3}\left(A_{2}^{T} Y B_{2}^{T}+C_{2}^{T} Z D_{2}^{T}\right) T_{4}$, where $Y \in R^{p \times q}, Z \in R^{s \times t}$ are arbitrary, or, especially, $X_{1}^{1}=0$ and $X_{2}^{1}=0$, then the solution pair $X_{1}^{k}, X_{2}^{k}$, generated by Algorithm 5 , is the least Frobenius norm solution of the matrix equations (1).

Suppose that Problem 1 is consistent. Obviously the solution set $S_{r}$ of (1) is nonempty. For given matrices pair $\widetilde{X}_{1} \in R^{k \times r}, \widetilde{X}_{2} \in R^{m \times n}$, we consider two case:

Case 1. Consider $\widetilde{X}_{1} \in R_{r}^{k \times r}\left(T_{1}, T_{2}\right), \widetilde{X}_{2} \in R_{r}^{m \times n}\left(T_{3}, T_{4}\right)$.

We have

$$
\begin{gathered}
A_{1} X_{1} B_{1}+A_{2} X_{2} B_{2}=E, \\
C_{1} X_{1} D_{1}+C_{2} X_{2} D_{2}=F, \\
\left\{\begin{array}{c}
A_{1}\left(X_{1}-\widetilde{X}_{1}\right) B_{1}+A_{2}\left(X_{2}-\widetilde{X}_{2}\right) B_{2} \\
=E-A_{1} \widetilde{X}_{1} B_{1}-A_{2} \widetilde{X}_{2} B_{2}, \\
C_{1}\left(X_{1}-\widetilde{X}_{1}\right) D_{1}+C_{2}\left(X_{2}-\widetilde{X}_{2}\right) D_{2} \\
=F-C_{1} \widetilde{X}_{1} D_{1}-C_{2} \widetilde{X}_{2} D_{2} .
\end{array}\right.
\end{gathered}
$$

Let $\bar{X}_{1}=X_{1}-\widetilde{X}_{1}, \bar{X}_{2}=X_{2}-\widetilde{X}_{2}, \bar{E}=E-A_{1} \widetilde{X}_{1} B_{1}-$ $A_{2} \widetilde{X}_{2} B_{2}$, and $\bar{F}=F-C_{1} \widetilde{X}_{1} D_{1}-C_{2} \widetilde{X}_{2} D_{2}$; then Problem 2 is equivalent to finding the least Frobenius norm solution pair of the system

$$
\begin{aligned}
& A_{1} \bar{X}_{1} B_{1}+A_{2} \bar{X}_{2} B_{2}=\bar{E} \\
& C_{1} \bar{X}_{1} D_{1}+C_{2} \bar{X}_{2} D_{2}=\bar{F}
\end{aligned}
$$

which can be obtained using Algorithm 5 with the initial matrix pair $X_{1}^{1}=A_{1}^{T} Y B_{1}^{T}+C_{1}^{T} Z D_{1}^{T}+T_{1}\left(A_{1}^{T} Y B_{1}^{T}+C_{1}^{T} Z D_{1}^{T}\right) T_{2}$,
$X_{2}^{1}=A_{2}^{T} Y B_{2}^{T}+C_{2}^{T} Z D_{2}^{T}+T_{3}\left(A_{2}^{T} Y B_{2}^{T}+C_{2}^{T} Z D_{2}^{T}\right) T_{4}$, where $Y \in R^{p \times q}, Z \in R^{s \times t}$ are arbitrary, or, especially, $\bar{X}_{1}^{1}=0$, $\bar{X}_{2}^{1}=0$, and the solution of the matrix optimal approximation Problem 2 can be represented as $\widehat{X}_{1}=\bar{X}_{1}^{k}+\widetilde{X}_{1}, \widehat{X}_{2}=\bar{X}_{2}^{k}+\widetilde{X}_{2}$.

Case 2. $\widetilde{X}_{1} \in R^{k \times r}, \widetilde{X}_{2} \in R^{m \times n}$, and $\widetilde{X}_{1}, \widetilde{X}_{2}$ are not generalized reflexive matrices.

Lemma 10. Suppose $H_{1} \in R_{r}^{k \times r}\left(T_{1}, T_{2}\right), H_{2} \in R_{a}^{m \times n}\left(T_{1}, T_{2}\right)$; then $\operatorname{tr}\left(H_{1}^{T} H_{2}\right)=0$.

Proof. Since $H_{1}=T_{1} H_{1} T_{2}, H_{2}=-T_{1} H_{2} T_{2}$, we have

$$
\begin{aligned}
\operatorname{tr}\left(H_{1}^{T} H_{2}\right) & =\operatorname{tr}\left[\left(T_{1} H_{1} T_{2}\right)^{T}\left(-T_{1} H_{2} T_{2}\right)\right] \\
& =-\operatorname{tr}\left(T_{2}^{T} H_{1}^{T} T_{1}^{T} T_{1} H_{2} T_{2}\right) \\
& =-\operatorname{tr}\left(T_{2}^{T} H_{1}^{T} T_{1}^{T} T_{1} H_{2} T_{2}\right)=-\operatorname{tr}\left(T_{2}^{T} H_{1}^{T} H_{2} T_{2}\right) \\
& =-\operatorname{tr}\left(H_{1}^{T} H_{2} T_{2} T_{2}^{T}\right)=-\operatorname{tr}\left(H_{1}^{T} H_{2}\right) .
\end{aligned}
$$

So, $\operatorname{tr}\left(H_{1}^{T} H_{2}\right)=0$.

For $\left\{X_{1}, X_{2}\right\} \in S_{r}$, by Lemma 10, we have

$$
\begin{aligned}
\left\|X_{1}-\widetilde{X}_{1}\right\|^{2} & =\left\|X_{1}-\left(\frac{\widetilde{X}_{1}+T_{1} \widetilde{X}_{1} T_{2}}{2}+\frac{\widetilde{X}_{1}-T_{1} \widetilde{X}_{1} T_{2}}{2}\right)\right\|^{2} \\
& =\left\|\left(X_{1}-\frac{\widetilde{X}_{1}+T_{1} \widetilde{X}_{1} T_{2}}{2}\right)-\frac{\widetilde{X}_{1}-T_{1} \widetilde{X}_{1} T_{2}}{2}\right\|^{2} \\
& =\left\|X_{1}-\frac{\widetilde{X}_{1}+T_{1} \widetilde{X}_{1} T_{2}}{2}\right\|^{2}+\left\|\frac{\widetilde{X}_{1}-T_{1} \widetilde{X}_{1} T_{2}}{2}\right\|^{2},
\end{aligned}
$$




$$
\begin{aligned}
\left\|X_{2}-\widetilde{X}_{2}\right\|^{2} & =\left\|X_{2}-\left(\frac{\widetilde{X}_{2}+T_{3} \widetilde{X}_{2} T_{4}}{2}+\frac{\widetilde{X}_{2}-T_{3} \widetilde{X}_{2} T_{4}}{2}\right)\right\|^{2} \\
& =\left\|\left(X_{2}-\frac{\widetilde{X}_{2}+T_{3} \widetilde{X}_{2} T_{4}}{2}\right)-\frac{\widetilde{X}_{2}-T_{3} \widetilde{X}_{2} T_{4}}{2}\right\|^{2} \\
& =\left\|X_{2}-\frac{\widetilde{X}_{2}+T_{3} \widetilde{X}_{2} T_{4}}{2}\right\|^{2}+\left\|\frac{\widetilde{X}_{2}-T_{3} \widetilde{X}_{2} T_{4}}{2}\right\|^{2} .
\end{aligned}
$$

For any $\left\{X_{1}, X_{2}\right\} \in S_{r}$, we have

$$
\begin{aligned}
& \left\|\widehat{X}_{1}-\widetilde{X}_{1}\right\|^{2}+\left\|\widehat{X}_{2}-\widetilde{X}_{2}\right\|^{2} \\
& =\min _{\left\{X_{1}, X_{2}\right\} \in S_{r}}\left(\left\|X_{1}-\widetilde{X}_{1}\right\|^{2}+\left\|X_{2}-\widetilde{X}_{2}\right\|^{2}\right) \\
& =\left\|\frac{\widetilde{X}_{1}-T_{1} \widetilde{X}_{1} T_{2}}{2}\right\|^{2}+\left\|\frac{\widetilde{X}_{2}-T_{3} \widetilde{X}_{2} T_{4}}{2}\right\|^{2} \\
& +\min _{\left\{X_{1}, X_{2}\right\} \in S_{r}}\left(\left\|X_{1}-\frac{\widetilde{X}_{1}+T_{1} \widetilde{X}_{1} T_{2}}{2}\right\|^{2}\right. \\
& \left.+\left\|X_{2}-\frac{\widetilde{X}_{2}+T_{3} \widetilde{X}_{2} T_{4}}{2}\right\|^{2}\right) .
\end{aligned}
$$

That is, Problem 2 is equivalent to finding $\left\{\widehat{X}_{1}, \widehat{X}_{2}\right\} \in S_{r}$ such that $\left\|X_{1}-\left(\widetilde{X}_{1}+T_{1} \widetilde{X}_{1} T_{2}\right) / 2\right\|^{2}+\left\|X_{2}-\left(\widetilde{X}_{1}+T_{3} \widetilde{X}_{2} T_{4}\right) / 2\right\|^{2}$ reach a minimum.

For any $\left\{X_{1}, X_{2}\right\} \in S_{r}$, we have

$$
\begin{aligned}
& A_{1} X_{1} B_{1}+A_{2} X_{2} B_{2}=E \\
& C_{1} X_{1} D_{1}+C_{2} X_{2} D_{2}=F \\
& \quad\left\{\begin{array}{c}
A_{1}\left(X_{1}-\frac{\widetilde{X}_{1}+T_{1} \widetilde{X}_{1} T_{2}}{2}\right) B_{1} \\
\quad+A_{2}\left(X_{2}-\frac{\widetilde{X}_{2}+T_{3} \widetilde{X}_{2} T_{4}}{2}\right) B_{2} \\
=E-A_{1} \frac{\widetilde{X}_{1}+T_{1} \widetilde{X}_{1} T_{2}}{2} B_{1}-A_{2} \frac{\widetilde{X}_{2}+T_{3} \widetilde{X}_{2} T_{4}}{2} B_{2} \\
C_{1}\left(X_{1}-\frac{\widetilde{X}_{1}+T_{1} \widetilde{X}_{1} T_{2}}{2}\right) D_{1} \\
\quad+C_{2}\left(X_{2}-\frac{\widetilde{X}_{2}+T_{3} \widetilde{X}_{2} T_{4}}{2}\right) D_{2} \\
=F-C_{1} \frac{\widetilde{X}_{1}+T_{1} \widetilde{X}_{1} T_{2}}{2} D_{1}-C_{2} \frac{\widetilde{X}_{2}+T_{3} \widetilde{X}_{2} T_{4}}{2} D_{2} .
\end{array}\right.
\end{aligned}
$$

Let $\bar{X}_{1}=X_{1}-\left(\widetilde{X}_{1}+T_{1} \widetilde{X}_{1} T_{2}\right) / 2, \bar{X}_{2}=X_{2}-\left(\widetilde{X}_{2}+\right.$ $\left.T_{3} \widetilde{X}_{2} T_{4}\right) / 2, \bar{E}=E-A_{1}\left(\left(\widetilde{X}_{1}+T_{1} \widetilde{X}_{1} T_{2}\right) / 2\right) B_{1}-A_{2}\left(\left(\widetilde{X}_{2}+\right.\right.$ $\left.\left.T_{3} \widetilde{X}_{2} T_{4}\right) / 2\right) B_{2}$, and $\bar{F}=F-C_{1}\left(\left(\widetilde{X}_{1}+T_{1} \widetilde{X}_{1} T_{2}\right) / 2\right) D_{1}-C_{2}\left(\left(\widetilde{X}_{2}+\right.\right.$ $\left.\left.T_{3} \widetilde{X}_{2} T_{4}\right) / 2\right) D_{2}$ and then to finding $\left\{\widehat{X}_{1}, \widehat{X}_{2}\right\} \in S$ such that $\left\|X_{1}-\left(\widetilde{X}_{1}+T_{1} \widetilde{X}_{1} T_{2}\right) / 2\right\|^{2}+\left\|X_{2}-\left(\widetilde{X}_{2}+T_{3} \widetilde{X}_{2} T_{4}\right) / 2\right\|^{2}$ reach a minimum is equivalent to finding the least Frobenius norm generalized reflexive solution pair $\bar{X}_{1}^{k}, \bar{X}_{2}^{k}$ of the system

$$
\begin{aligned}
& A_{1} \bar{X}_{1} B_{1}+A_{2} \bar{X}_{2} B_{2}=\bar{E} \\
& C_{1} \bar{X}_{1} D_{1}+C_{2} \bar{X}_{2} D_{2}=\bar{F},
\end{aligned}
$$

which can be obtained using Algorithm 5.

So we can have the least Frobenius norm generalized reflexive solution of Problem 2 as $\widehat{X}_{1}=\bar{X}_{1}^{k}+\left(\widetilde{X}_{1}+T_{1} \widetilde{X}_{1} T_{2}\right) / 2$, $\widehat{X}_{2}=\bar{X}_{2}^{k}+\left(\widetilde{X}_{2}+T_{3} \widetilde{X}_{2} T_{4}\right) / 2$.

\section{Iterative Algorithm for Solving Problems 3 and 4}

In this section, we present an iterative algorithm for solving Problems 3 and 4.

Algorithm 11. (1) Input matrices $A_{1} \in R^{p \times k}, A_{2} \in R^{p \times m}, B_{1} \in$ $R^{r \times q}, B_{2} \in R^{n \times q}, C_{1} \in R^{s \times k}, C_{2} \in R^{s \times m}, D_{1} \in R^{r \times t}, D_{2} \in R^{n \times t}$, $E \in R^{p \times q}, F \in R^{s \times t}, X_{1}^{1} \in R_{a}^{k \times r}$, and $X_{2}^{1} \in R_{a}^{m \times n}$ (where $X_{1}^{1}, X_{2}^{1}$ are any initial generalized antireflexive matrices).

(2) Calculate

$$
\begin{gathered}
E^{1}=E ; \quad F^{1}=F ; \\
Q_{1}^{1}=A_{1}^{T} E^{1} B_{1}^{T}+C_{1}^{T} F^{1} D_{1}^{T} ; \quad Q_{2}^{1}=A_{2}^{T} E^{1} B_{2}^{T}+C_{2}^{T} F^{1} D_{2}^{T} ; \\
P_{1}^{1}=Q_{1}^{1}-T_{1} Q_{1}^{1} T_{2} ; \quad P_{2}^{1}=Q_{2}^{1}-T_{3} Q_{2}^{1} T_{4} ; \\
\beta^{1}=\left(\operatorname{tr}\left[\left(E^{1}\right)^{T}\left(A_{1} P_{1}^{1} B_{1}+A_{2} P_{2}^{1} B_{2}\right)\right]\right. \\
\left.+\operatorname{tr}\left[\left(F^{1}\right)^{T}\left(C_{1} P_{1}^{1} D_{1}+C_{2} P_{2}^{1} D_{2}\right)\right]\right) \\
\times\left(\left\|A_{1} P_{1}^{1} B_{1}+A_{2} P_{2}^{1} B_{2}\right\|^{2}+\left\|C_{1} P_{1}^{1} D_{1}+C_{2} P_{2}^{1} D_{2}\right\|^{2}\right)^{-1} ; \\
\Delta X_{1}^{1}=\beta^{1} P_{1}^{1} ; \quad \Delta X_{2}^{1}=\beta^{1} P_{2}^{1} ; \quad k=1 .
\end{gathered}
$$

(3) If $\Delta X=\operatorname{diag}\left(\Delta X_{1}^{k}, \Delta X_{2}^{k}\right)=0(k=1,2, \ldots)$, then stop. Otherwise,

$$
\begin{aligned}
& X_{1}^{k+1}=X_{1}^{k}+\Delta X_{1}^{k} \\
& X_{2}^{k+1}=X_{2}^{k}+\Delta X_{2}^{k} .
\end{aligned}
$$

(4) Calculate

$$
\begin{gathered}
E^{k+1}=E^{k}-\left(A_{1} \Delta X_{1}^{k} B_{1}+A_{2} \Delta X_{2}^{k} B_{2}\right) \\
F^{k+1}=F^{k}-\left(C_{1} \Delta X_{1}^{k} D_{1}+C_{2} \Delta X_{2}^{k} D_{2}\right) ; \\
Q_{1}^{k+1}=A_{1}^{T} E^{k+1} B_{1}^{T}+C_{1}^{T} F^{k+1} D_{1}^{T}
\end{gathered}
$$




$$
\begin{gathered}
Q_{2}^{k+1}=A_{2}^{T} E^{k+1} B_{2}^{T}+C_{2}^{T} F^{k+1} D_{2}^{T} ; \\
P_{1}^{k+1}=Q_{1}^{k+1}-T_{1} Q_{1}^{k+1} T_{2} ; \quad P_{2}^{k+1}=Q_{2}^{k+1}-T_{3} Q_{2}^{k+1} T_{4} ; \\
\beta^{k+1}=\left(\operatorname{tr}\left[\left(E^{k+1}\right)^{T}\left(A_{1} P_{1}^{k+1} B_{1}+A_{2} P_{2}^{k+1} B_{2}\right)\right]\right. \\
\left.+\operatorname{tr}\left[\left(F^{k+1}\right)^{T}\left(C_{1} P_{1}^{k+1} D_{1}+C_{2} P_{2}^{k+1} D_{2}\right)\right]\right) \\
\times\left(\left\|A_{1} P_{1}^{k+1} B_{1}+A_{2} P_{2}^{k+1} B_{2}\right\|^{2}\right. \\
\left.+\left\|C_{1} P_{1}^{k+1} D_{1}+C_{2} P_{2}^{k+1} D_{2}\right\|^{2}\right)^{-1} ; \\
\Delta X_{1}^{k+1}=\beta^{k+1} P_{1}^{k+1} ; \quad \Delta X_{2}^{k+1}=\beta^{k+1} P_{2}^{k+1} ; \quad k=k+1 .
\end{gathered}
$$

Go to (3).

The properties of Algorithm 11 can be proposed similarly to Algorithm 5.

\section{Examples}

In this section, we show two numerical examples to illustrate the efficiency of Algorithms 5 and 11. All computations are performed by MATLAB 7. For the influence of the error of calculation, we consider the matrix $R$ as a zero matrix if $\|R\|<$ $10^{-10}$.

Example 12. Consider the generalized reflexive solution of the linear matrix equations:

$$
\begin{aligned}
& A_{1} X_{1} B_{1}+A_{2} X_{2} B_{2}=E, \\
& C_{1} X_{1} D_{1}+C_{2} X_{2} D_{2}=F,
\end{aligned}
$$

where

$$
\begin{aligned}
& A_{1}=\left(\begin{array}{ccc}
139 & 105 & 54 \\
124 & 176 & 50 \\
159 & 35 & 175 \\
191 & 196 & 147
\end{array}\right), \\
& B_{1}=\left(\begin{array}{ccccc}
13 & 117 & 103 & 87 & 116 \\
198 & 85 & 67 & 45 & 152
\end{array}\right) \text {, } \\
& A_{2}=\left(\begin{array}{cc}
27 & 60 \\
2 & 132 \\
179 & 57 \\
40 & 94
\end{array}\right), \\
& B_{2}=\left(\begin{array}{ccccc}
106 & 76 & 92 & 12 & 83 \\
128 & 157 & 114 & 121 & 61 \\
42 & 136 & 159 & 10 & 175
\end{array}\right), \\
& C_{1}=\left(\begin{array}{ccc}
3 & 88 & 192 \\
154 & 100 & 145 \\
194 & 43 & 82 \\
198 & 129 & 149 \\
158 & 64 & 54
\end{array}\right), \quad D_{1}=\left(\begin{array}{ccc}
88 & 31 & 140 \\
71 & 135 & 146
\end{array}\right)
\end{aligned}
$$

$$
\begin{array}{cc}
C_{2}=\left(\begin{array}{cc}
4 & 63 \\
115 & 3 \\
90 & 77 \\
9 & 137 \\
5 & 19
\end{array}\right), & D_{2}=\left(\begin{array}{ccc}
96 & 90 & 55 \\
111 & 143 & 51 \\
24 & 179 & 173
\end{array}\right), \\
T_{1}=\left(\begin{array}{ccc}
-1 & 0 & 0 \\
0 & 0 & 1 \\
0 & 1 & 0
\end{array}\right), \quad T_{2}=\left(\begin{array}{cc}
0 & -1 \\
-1 & 0
\end{array}\right), \\
T_{3}=\left(\begin{array}{ll}
0 & 1 \\
1 & 0
\end{array}\right), \quad T_{4}=\left(\begin{array}{ccc}
0 & 1 & 0 \\
1 & 0 & 0 \\
0 & 0 & -1
\end{array}\right),
\end{array}
$$

$E=\left(\begin{array}{lllll}4079684 & 5931341 & 5257546 & 3760503 & 5978636 \\ 4507138 & 7309406 & 6800596 & 4204046 & 7209376 \\ 5983467 & 8290427 & 6916092 & 5829089 & 5964332 \\ 4470189 & 8583167 & 7694045 & 5535093 & 8046508\end{array}\right)$,

$$
F=\left(\begin{array}{ccc}
1148659 & -415508 & 175592 \\
5221721 & 3459626 & 5372914 \\
6867542 & 6499561 & 7947258 \\
6828512 & 5933355 & 8858444 \\
3684631 & 3115265 & 5873214
\end{array}\right) .
$$

Let

$$
X_{1}^{1}=\left(\begin{array}{ll}
0 & 0 \\
0 & 0 \\
0 & 0
\end{array}\right), \quad X_{2}^{1}=\left(\begin{array}{lll}
0 & 0 & 0 \\
0 & 0 & 0
\end{array}\right) .
$$

Using Algorithm 5 and iterating 1214 steps, we obtain the least Frobenius norm solution pair of the matrix equation in Example 12 as follows:

$$
\begin{gathered}
X_{1}=\left(\begin{array}{cc}
122.0000 & 122.0000 \\
86.0000 & -29.0000 \\
29.0000 & -86.0000
\end{array}\right), \\
X_{2}=\left(\begin{array}{ccc}
57.0000 & 126.0000 & -35.0000 \\
126.0000 & 57.0000 & 35.0000
\end{array}\right) .
\end{gathered}
$$

Example 13. Consider the generalized antireflexive solution of the linear matrix equations:

$$
A_{1} X_{1} B_{1}+A_{2} X_{2} B_{2}=E, \quad C_{1} X_{1} D_{1}+C_{2} X_{2} D_{2}=F,
$$

where $A_{1}, B_{1}, A_{2}, B_{2}, C_{1}, D_{1}, C_{2}, D_{2}, T_{1}, T_{2}, T_{3}$, and $T_{4}$ are the same matrices of Example 12:

$$
\begin{gathered}
E=\left(\begin{array}{ccccc}
-743633 & 6710933 & 7426284 & 2563311 & 8338860 \\
1535166 & 7945314 & 9163408 & 1986230 & 12338200 \\
982308 & 14732018 & 17074320 & 3775802 & 18238112 \\
1999993 & 12662336 & 13364050 & 5402942 & 16219108
\end{array}\right), \\
F=\left(\begin{array}{rrrr}
5228109 & 6926269 & 12710902 \\
7508081 & 7671994 & 14810466 \\
4201528 & 4853203 & 12104012 \\
5302290 & 5127440 & 15650036 \\
2867500 & -410942 & 5039552
\end{array}\right) .
\end{gathered}
$$


Let

$$
X_{1}^{1}=\left(\begin{array}{ll}
0 & 0 \\
0 & 0 \\
0 & 0
\end{array}\right), \quad X_{2}^{1}=\left(\begin{array}{lll}
0 & 0 & 0 \\
0 & 0 & 0
\end{array}\right)
$$

Using Algorithm 11 and iterating 240 steps, we obtain the least Frobenius norm solution pair of the matrix equation in Example 13 as follows:

$$
\begin{gathered}
X_{1}=\left(\begin{array}{cc}
226.0000 & -226.0000 \\
59.0000 & 191.0000 \\
191.0000 & 59.0000
\end{array}\right), \\
X_{2}=\left(\begin{array}{ccc}
189.0000 & -63.0000 & 268.0000 \\
63.0000 & -189.0000 & 268.0000
\end{array}\right) .
\end{gathered}
$$

The numbers of the parameter matrices $A_{1}, A_{2}, B_{1}, B_{2}$, $C_{1}, C_{2}, D_{1}$, and $D_{2}$ in our examples are larger than the numbers of the parameter matrices in the example of [31]. To our examples, the algorithm of [31] is not convergent. The numerical examples demonstrate that our algorithm has merits of good numerical stability and ease to program.

\section{Conflict of Interests}

The authors declare that there is no conflict of interests regarding the publication of this paper.

\section{Acknowledgments}

This research was supported by Grants from the Key Project of Scientific Research Innovation Foundation of Shanghai Municipal Education Commission (13ZZ080), the National Natural Science Foundation of China (11171205), the Natural Science Foundation of Shanghai (11ZR1412500), and the Nature Science Foundation of Anhui Provincial Education (ky2008b253, KJ2013A248).

\section{References}

[1] T. Meng, "Experimental design and decision support," in Expert Systems: The Technology of Knowledge Management and Decision Making for the 21st Century, C. Leondes, Ed., vol. 1, p. 119, Academic Press, New York, NY, USA, 2001.

[2] M. Dehghan and M. Hajarian, "An iterative algorithm for solving a pair of matrix equations $A Y B=E, C Y D=F$ over generalized centro-symmetric matrices," Computers \& Mathematics with Applications, vol. 56, no. 12, pp. 3246-3260, 2008.

[3] M. Dehghan and M. Hajarian, "An iterative algorithm for the reflexive solutions of the generalized coupled Sylvester matrix equations and its optimal approximation," Applied Mathematics and Computation, vol. 202, no. 2, pp. 571-588, 2008.

[4] A. L. Andrew, "Solution of equations involving centrosymmetric matrices," Technometrics, vol. 15, no. 2, pp. 405-407, 1973.

[5] A. Navarra, P. L. Odell, and D. M. Young, "Representation of the general common solution to the matrix equations $A_{1} X B_{1}=C_{1}$ and $A_{2} X B_{2}=C_{2}$ with applications," Computers \& Mathematics with Applications, vol. 41, no. 7-8, pp. 929-935, 2001.
[6] Z.-H. Peng, X.-Y. Hu, and L. Zhang, "An efficient algorithm for the least-squares reflexive solution of the matrix equation $A_{1} X B_{1}=C_{1}, A_{2} X B_{2}=C_{2}$," Applied Mathematics and Computation, vol. 181, no. 2, pp. 988-999, 2006.

[7] X. Sheng and G. Chen, "A finite iterative method for solving a pair of linear matrix equations $(A X B, C X D)=(E, F)$, , Applied Mathematics and Computation, vol. 189, no. 2, pp. 1350-1358, 2007.

[8] A.-G. Wu, G. Feng, G.-R. Duan, and W.-J. Wu, "Finite iterative solutions to a class of complex matrix equations with conjugate and transpose of the unknowns," Mathematical and Computer Modelling, vol. 52, no. 9-10, pp. 1463-1478, 2010.

[9] A.-G. Wu, G. Feng, G.-R. Duan, and W.-J. Wu, "Iterative solutions to coupled Sylvester-conjugate matrix equations," Computers \& Mathematics with Applications, vol. 60, no. 1, pp. 54-66, 2010.

[10] A.-G. Wu, B. Li, Y. Zhang, and G.-R. Duan, "Finite iterative solutions to coupled Sylvester-conjugate matrix equations," Applied Mathematical Modelling, vol. 35, no. 3, pp. 1065-1080, 2011.

[11] Y. X. Yuan, "Least squares solutions of matrix equation $A X B=$ $E, C X D=F$," Journal of East China Shipbuilding Institute, vol. 18, no. 3, pp. 29-31, 2004.

[12] Y.-X. Peng, X.-Y. Hu, and L. Zhang, "An iterative method for symmetric solutions and optimal approximation solution of the system of matrix equations $A_{1} X B_{1}=C_{1}, A_{2} X B_{2}=C_{2}$," Applied Mathematics and Computation, vol. 183, no. 2, pp. 1127-1137, 2006.

[13] S. K. Mitra, "Common solutions to a pair of linear matrix equations $A_{1} X B_{1}=C_{1}$ and $A_{2} X B_{2}=C_{2}$," Cambridge Philosophical Society, vol. 74, no. 2, pp. 213-216, 1973.

[14] N. Shinozaki and M. Sibuya, "Consistency of a pair of matrix equations with an application," Keio Science and Technology Reports, vol. 27, no. 10, pp. 141-146, 1974.

[15] J. W. van der Woude, Freeback decoupling and stabilization for linear systems with multiple exogenous variables [Ph.D. thesis], Technical University of Eindhoven, Eindhoven, The Netherlands, 1987.

[16] Q.-W. Wang, "A system of matrix equations and a linear matrix equation over arbitrary regular rings with identity," Linear Algebra and its Applications, vol. 384, no. 1-3, pp. 43-54, 2004.

[17] M. Dehghan and M. Hajarian, "The reflexive and anti-reflexive solutions of a linear matrix equation and systems of matrix equations," The Rocky Mountain Journal of Mathematics, vol. 40, no. 3, pp. 825-848, 2010.

[18] Q.-W. Wang, J.-H. Sun, and S.-Z. Li, "Consistency for bi(skew)symmetric solutions to systems of generalized Sylvester equations over a finite central algebra," Linear Algebra and its Applications, vol. 353, no. 1-3, pp. 169-182, 2002.

[19] Q.-W. Wang, "Bisymmetric and centrosymmetric solutions to systems of real quaternion matrix equations," Computers \& Mathematics with Applications, vol. 49, no. 5-6, pp. 641-650, 2005.

[20] F. Ding, P. X. Liu, and J. Ding, "Iterative solutions of the generalized Sylvester matrix equations by using the hierarchical identification principle," Applied Mathematics and Computation, vol. 197, no. 1, pp. 41-50, 2008.

[21] L. Xie, J. Ding, and F. Ding, "Gradient based iterative solutions for general linear matrix equations," Computers \& Mathematics with Applications, vol. 58, no. 7, pp. 1441-1448, 2009. 
[22] L. Xie, Y. Liu, and H. Yang, "Gradient based and least squares based iterative algorithms for matrix equations $A X B+C X^{T} D=$ F," Applied Mathematics and Computation, vol. 217, no. 5, pp. 2191-2199, 2010.

[23] J. Ding, Y. Liu, and F. Ding, "Iterative solutions to matrix equations of the form $A_{i} X B_{i}=F_{i}$," Computers \& Mathematics with Applications, vol. 59, no. 11, pp. 3500-3507, 2010.

[24] B. Zhou, J. Lam, and G.-R. Duan, "Gradient-based maximal convergence rate iterative method for solving linear matrix equations," International Journal of Computer Mathematics, vol. 87, no. 3, pp. 515-527, 2010.

[25] Z.-Y. Li, B. Zhou, Y. Wang, and G.-R. Duan, "Numerical solution to linear matrix equation by finite steps iteration," IET Control Theory \& Applications, vol. 4, no. 7, pp. 1245-1253, 2010.

[26] Z.-Y. Li, Y. Wang, B. Zhou, and G.-R. Duan, "Least squares solution with the minimum-norm to general matrix equations via iteration," Applied Mathematics and Computation, vol. 215, no. 10 , pp. 3547-3562, 2010.

[27] B. Zhou, J. Lam, and G.-R. Duan, "On Smith-type iterative algorithms for the Stein matrix equation," Applied Mathematics Letters, vol. 22, no. 7, pp. 1038-1044, 2009.

[28] Y.-B. Deng, Z.-Z. Bai, and Y.-H. Gao, "Iterative orthogonal direction methods for Hermitian minimum norm solutions of two consistent matrix equations," Numerical Linear Algebra with Applications, vol. 13, no. 10, pp. 801-823, 2006.

[29] Y.-T. Li and W.-J. Wu, "Symmetric and skew-antisymmetric solutions to systems of real quaternion matrix equations," Computers \& Mathematics with Applications, vol. 55, no. 6, pp. 1142-1147, 2008.

[30] M. Dehghan and M. Hajarian, "An efficient algorithm for solving general coupled matrix equations and its application," Mathematical and Computer Modelling, vol. 51, no. 9-10, pp. 1118-1134, 2010.

[31] M. Dehghan and M. Hajarian, "On the reflexive and antireflexive solutions of the generalised coupled Sylvester matrix equations," International Journal of Systems Science, vol. 41, no. 6, pp. 607-625, 2010.

[32] M. Dehghan and M. Hajarian, "The general coupled matrix equations over generalized bisymmetric matrices," Linear Algebra and its Applications, vol. 432, no. 6, pp. 1531-1552, 2010.

[33] B. Zhou, Z.-Y. Li, G.-R. Duan, and Y. Wang, "Weighted least squares solutions to general coupled Sylvester matrix equations," Journal of Computational and Applied Mathematics, vol. 224, no. 2, pp. 759-776, 2009.

[34] I. Jonsson and B. Kågström, "Recursive blocked algorithms for solving triangular systems-part I: one-sided and coupled Sylvester-type matrix equations," ACM Transactions on Mathematical Software, vol. 28, no. 4, pp. 392-415, 2002.

[35] B. Zhou, G.-R. Duan, and Z.-Y. Li, "Gradient based iterative algorithm for solving coupled matrix equations," Systems and Control Letters, vol. 58, no. 5, pp. 327-333, 2009.

[36] I. Jonsson and B. Kågström, "Recursive blocked algorithms for solving triangular systems-part II: two-sided and generalized Sylvester and Lyapunov matrix equations," ACM Transactions on Mathematical Software, vol. 28, no. 4, pp. 416-435, 2002.

[37] A.-P. Liao and Y. Lei, "Least-squares solution with the minimum-norm for the matrix equation $(A X B, G X H)=$ (C, D)," Computers \& Mathematics with Applications, vol. 50, no. 3-4, pp. 539-549, 2005.

[38] J. Cai and G. Chen, "An iterative algorithm for the least squares bisymmetric solutions of the matrix equations $A_{1} X B_{1}=C_{1}$,
$A_{2} \mathrm{XB}_{2}=C_{2}$," Mathematical and Computer Modelling, vol. 50, no. 7-8, pp. 1237-1244, 2009.

[39] F. Yin and G.-X. Huang, "An iterative algorithm for the least squares generalized reflexive solutions of the matrix equations $A X B=E, C X D=F$, Abstract and Applied Analysis, vol. 2012, Article ID 857284, 18 pages, 2012.

[40] Y. Lin and Q. W. Wang, "Iterative solution to a system of matrix equations," Abstract and Applied Analysis, vol. 2013, Article ID 124979, 7 pages, 2013.

[41] Y.-X. Peng, X.-Y. Hu, and L. Zhang, "An iteration method for the symmetric solutions and the optimal approximation solution of the matrix equation $A X B=C$, Applied Mathematics and Computation, vol. 160, no. 3, pp. 763-777, 2005. 


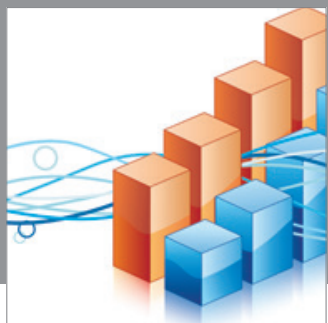

Advances in

Operations Research

mansans

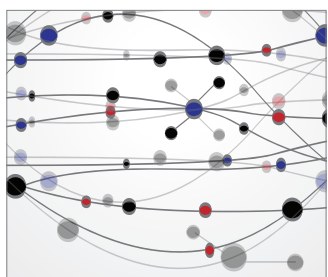

The Scientific World Journal
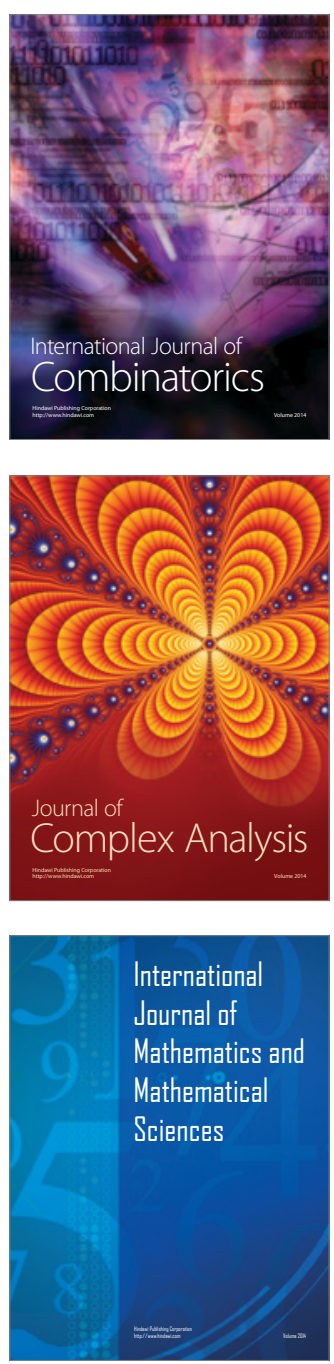
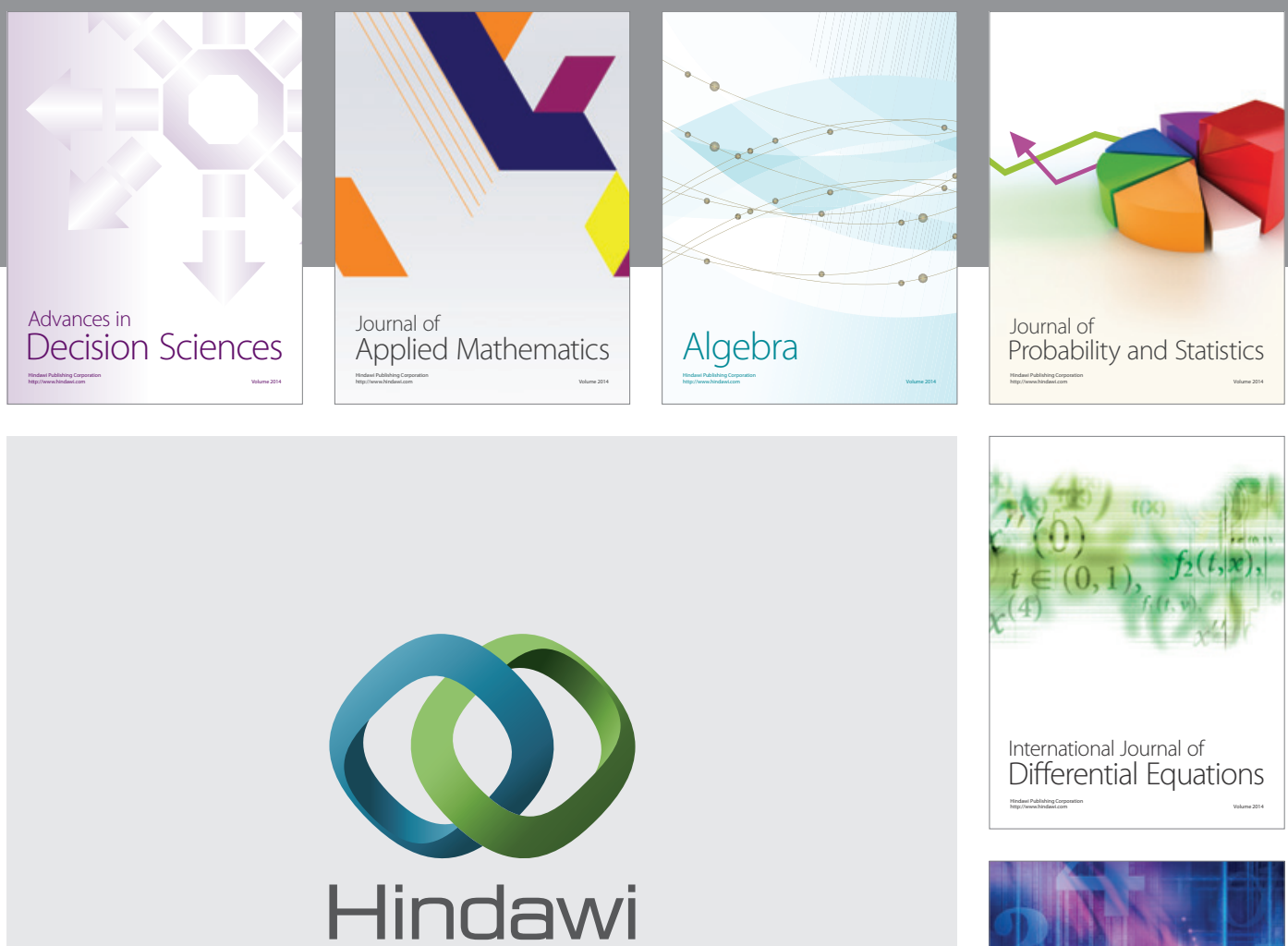

Submit your manuscripts at http://www.hindawi.com
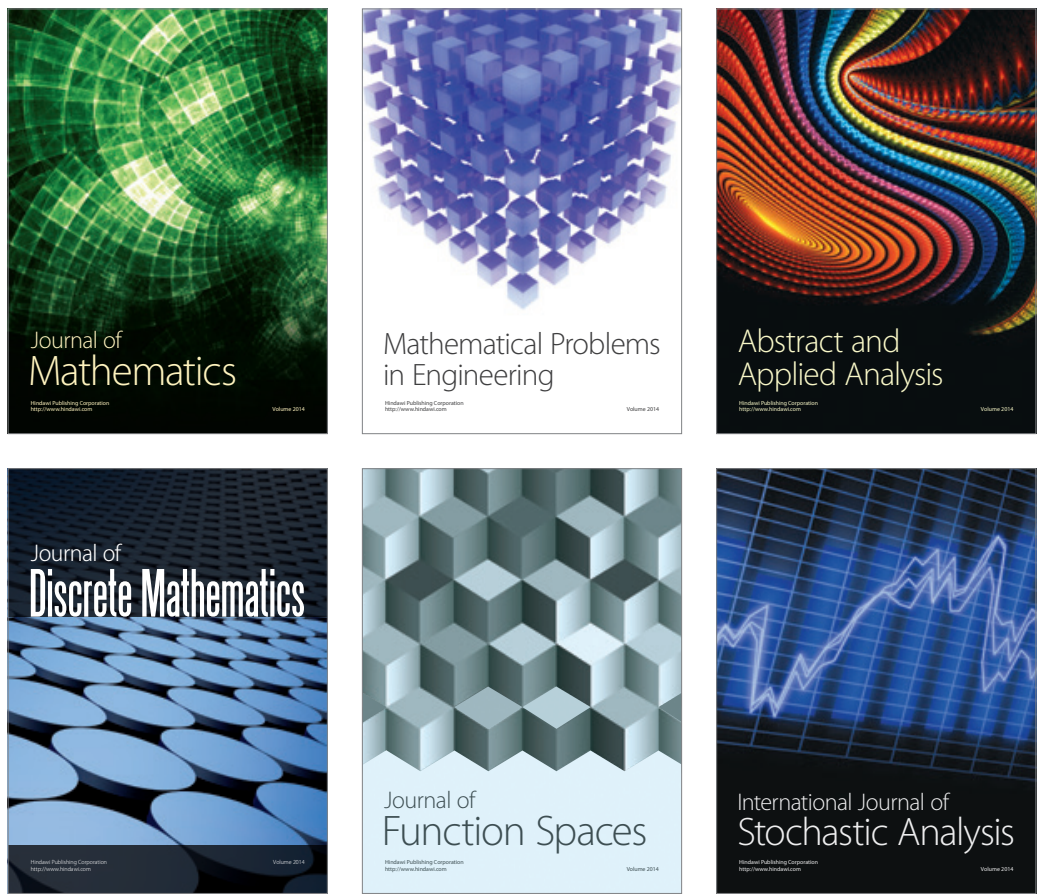

Journal of

Function Spaces

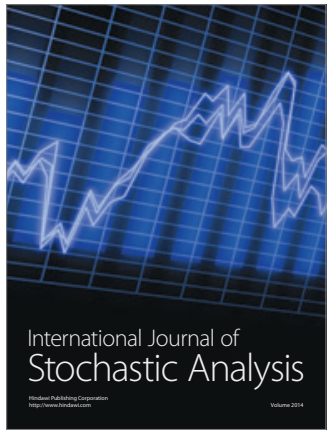

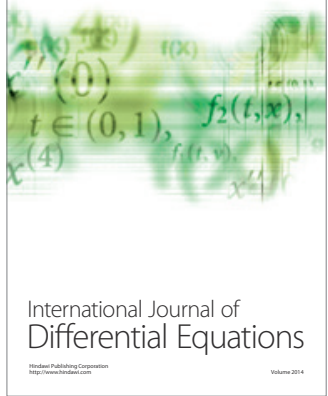
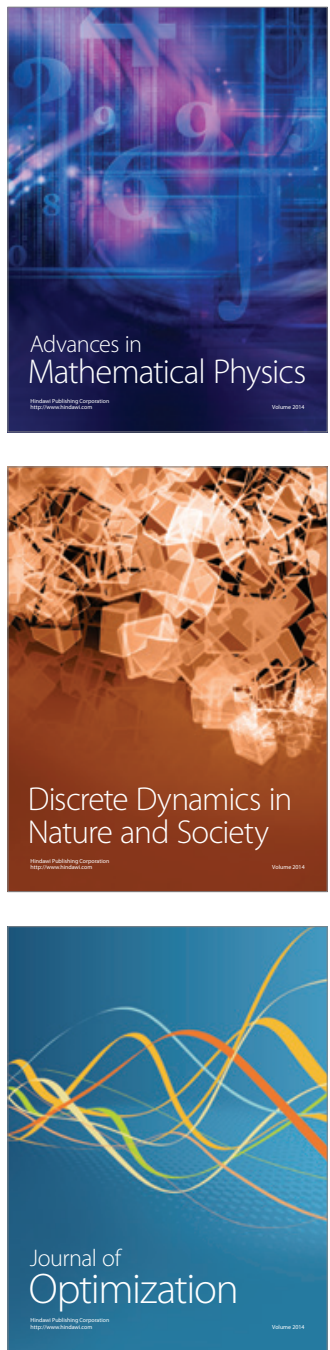